\title{
Chebyshev Polynomials and Continued Fractions Related
}

\author{
Jerzy Szczepański
}

Faculty of Mathematics and Computer Science, Jagiellonian University, Kraków

\section{Article history:}

Received 21 December 2019

Received in revised form

28 December 2019

Accepted 29 December 2019

Available online 31 December 2019

\begin{abstract}
Let $p, q$ be complex polynomials, $\operatorname{deg} p>\operatorname{deg} q \geq 0$. We consider the family of polynomials defined by the recurrence $P_{n+1}=2 p P_{n}-q P_{n-1}$ for $n=1,2,3, \ldots$ with arbitrary

$\mathrm{P}_{1}$ and $\mathrm{P}_{0}$ as well as the domain of the convergence of the infinite continued fraction
\end{abstract}

$$
f(z)=2 p(z)-\frac{q(z)}{2 p(z)-\frac{q(z)}{2 p(z)-\ldots}} .
$$

Key words: Chebyshev polynomials, continued fractions, Binet formula, Cassini identity

\section{Some polynomials of the Chebyshev type}

Let $P_{0}$ and $P_{1}$ be polynomials of one complex variable, $\operatorname{deg} P_{1}>\operatorname{deg} P_{0} \geq 0$. Let $p, q$ be polynomials of one complex variable, $\operatorname{deg} p>\operatorname{deg} q \geq 0, q \neq 0$. Define the family of polynomials $P_{n}$ by the recurrence formula

$$
P_{n+1}(z)=2 p(z) P_{n}(z)-q(z) P_{n-1}(z), \quad n=1,2,3, \ldots
$$

Note that (1) gives the Chebyshev polynomials of

- the first kind $T_{n}$ for $P_{0}(z)=1, P_{1}(z)=z, p(z)=z$ and $q(z)=1$

- the second kind $U_{n}$ for $P_{0}(z)=1, P_{1}(z)=2 z, p(z)=z$ and $q(z)=1$

- the third kind $V_{n}$ for $P_{0}(z)=1, P_{1}(z)=2 z-1, p(z)=z$ and $q(z)=1$

- the fourth kind $W_{n}$ for $P_{0}(z)=1, P_{1}(z)=2 z+1, p(z)=z$ and $q(z)=1$.

(See [2], Appendix B, Table B.2).

We write the recurrence (1) in the matrix form

$$
\left[\begin{array}{cc}
P_{n+1} & P_{n} \\
P_{n} & P_{n-1}
\end{array}\right]=\left[\begin{array}{cc}
2 p & -q \\
1 & 0
\end{array}\right]\left[\begin{array}{cc}
P_{n} & P_{n-1} \\
P_{n-1} & P_{n-2}
\end{array}\right]
$$

proceeding as in [1], p.80, where the Fibonacci sequence was considered, defined by the similar recurrence $\left[\begin{array}{cc}F_{n+1} & F_{n} \\ F_{n} & F_{n-1}\end{array}\right]=\left[\begin{array}{ll}1 & 1 \\ 1 & 0\end{array}\right]\left[\begin{array}{cc}F_{n} & F_{n-1} \\ F_{n-1} & F_{n-2}\end{array}\right]$ with $F_{0}=0$ and $F_{1}=1$.

Note that the characteristic polynomial

$$
w(\lambda)=\operatorname{det}\left[\begin{array}{cc}
2 p-\lambda & -q \\
1 & -\lambda
\end{array}\right]=(\lambda-p)^{2}-p^{2}+q
$$


of the matrix

$$
\left[\begin{array}{cc}
2 p & -q \\
1 & 0
\end{array}\right]
$$

addmits two different roots

$$
\lambda_{1}=p+\sqrt{p^{2}-q} \text { and } \lambda_{2}=p-\sqrt{p^{2}-q},
$$

as the polynomial $q$ is assumed to be nonzero.

Theorem 1.1 For the polynomial $P_{n}$ defined by (1) we get the following formula

$$
P_{n}=\frac{1}{\lambda_{1}-\lambda_{2}}\left[\left(\lambda_{1}^{n}-\lambda_{2}^{n}\right) P_{1}-\lambda_{1} \lambda_{2}\left(\lambda_{1}^{n-1}-\lambda_{2}^{n-1}\right) P_{0}\right]
$$

where $\lambda_{1}$ and $\lambda_{2}$ are the eigenvalues (4) of the matrix (3).

Proof. By the Jordan decomposition of the matrix (3) we get

$$
\left[\begin{array}{cc}
2 p & -q \\
1 & 0
\end{array}\right]=\frac{1}{\lambda_{1}-\lambda_{2}}\left[\begin{array}{cc}
\lambda_{1} & \lambda_{2} \\
1 & 1
\end{array}\right]\left[\begin{array}{cc}
\lambda_{1} & 0 \\
0 & \lambda_{2}
\end{array}\right]\left[\begin{array}{cc}
1 & -\lambda_{2} \\
-1 & \lambda_{1}
\end{array}\right]
$$

The $n$-th power of the matrix (3) equals

$$
\left[\begin{array}{cc}
2 p & -q \\
1 & 0
\end{array}\right]^{n}=\frac{1}{\lambda_{1}-\lambda_{2}}\left[\begin{array}{cc}
\lambda_{1} & \lambda_{2} \\
1 & 1
\end{array}\right]\left[\begin{array}{cc}
\lambda_{1}^{n} & 0 \\
0 & \lambda_{2}^{n}
\end{array}\right]\left[\begin{array}{cc}
1 & -\lambda_{2} \\
-1 & \lambda_{1}
\end{array}\right]
$$

Hence, by the recurrence

$$
\begin{aligned}
{\left[\begin{array}{cc}
P_{n+1} & P_{n} \\
P_{n} & P_{n-1}
\end{array}\right] } & =\left[\begin{array}{cc}
2 p & -q \\
1 & 0
\end{array}\right]\left[\begin{array}{cc}
P_{n} & P_{n-1} \\
P_{n-1} & P_{n-2}
\end{array}\right]=\left[\begin{array}{cc}
2 p & -q \\
1 & 0
\end{array}\right]^{2}\left[\begin{array}{cc}
P_{n-1} & P_{n-2} \\
P_{n-2} & P_{n-3}
\end{array}\right]=\ldots \\
& =\left[\begin{array}{cc}
2 p & -q \\
1 & 0
\end{array}\right]^{n-1}\left[\begin{array}{ll}
P_{2} & P_{1} \\
P_{1} & P_{0}
\end{array}\right]
\end{aligned}
$$

we obtain

$$
\left[\begin{array}{cc}
P_{n+1} & P_{n} \\
P_{n} & P_{n-1}
\end{array}\right]=\frac{1}{\lambda_{1}-\lambda_{2}}\left[\begin{array}{cc}
\lambda_{1} & \lambda_{2} \\
1 & 1
\end{array}\right]\left[\begin{array}{cc}
\lambda_{1}^{n-1} & 0 \\
0 & \lambda_{2}^{n-1}
\end{array}\right]\left[\begin{array}{cc}
1 & -\lambda_{2} \\
-1 & \lambda_{1}
\end{array}\right]\left[\begin{array}{cc}
P_{2} & P_{1} \\
P_{1} & P_{0}
\end{array}\right]
$$

Multiplying the above matrices we get

$$
P_{n}=\frac{1}{\lambda_{1}-\lambda_{2}}\left[\left(\lambda_{1}^{n}-\lambda_{2}^{n}\right) P_{1}-\lambda_{1} \lambda_{2}\left(\lambda_{1}^{n-1}-\lambda_{2}^{n-1}\right) P_{0}\right]
$$

Note that (5) corresponds to the well known Binet formula for the Fibonacci sequence

$$
F_{n}=\frac{\mu_{1}^{n}-\mu_{2}^{n}}{\mu_{1}-\mu_{2}}=\frac{1}{\sqrt{5}}\left[\left(\frac{\sqrt{5}+1}{2}\right)^{n}-\left(\frac{-\sqrt{5}+1}{2}\right)^{n}\right]
$$


where $\mu_{1}=\frac{\sqrt{5}+1}{2}$ and $\mu_{2}=\frac{-\sqrt{5}+1}{2}$ are eigenvalues of the matrix $\left[\begin{array}{ll}1 & 1 \\ 1 & 0\end{array}\right]$ that defines the Fibonacci sequence $\left[\begin{array}{cc}F_{n+1} & F_{n} \\ F_{n} & F_{n-1}\end{array}\right]=\left[\begin{array}{ll}1 & 1 \\ 1 & 0\end{array}\right]\left[\begin{array}{cc}F_{n} & F_{n-1} \\ F_{n-1} & F_{n-2}\end{array}\right]$ with $F_{0}=0$ and $F_{1}=1$.

Remark 1.2 The formula (5) works well with two known formulae (see [2] 1.49 and 1.52) for the Chebyshev polynomials of the first kind $T_{0}(x)=1, T_{1}(x)=x, T_{n+1}(x)=2 x T_{n}(x)-$ $T_{n-1}(x)$ if we put $p(x)=x, q(x)=1, \lambda_{1}(x)=x+\sqrt{x^{2}-1}, \lambda_{2}(x)=x-\sqrt{x^{2}-1}, P_{0}(x)=1$ and $P_{1}(x)=x$ :

$$
\begin{aligned}
T_{n}(x) & =\frac{1}{\lambda_{1}-\lambda_{2}}\left[\left(\lambda_{1}^{n}-\lambda_{2}^{n}\right) x-\left(\lambda_{1}^{n-1}-\lambda_{2}^{n-1}\right)\right] \\
& =\frac{1}{\lambda_{1}-\lambda_{2}}\left[\lambda_{1}^{n}\left(x-\frac{1}{\lambda_{1}}\right)-\lambda_{2}^{n}\left(x-\frac{1}{\lambda_{2}}\right)\right] \\
& =\frac{1}{2}\left(\lambda_{1}^{n}+\lambda_{2}^{n}\right) \\
& =\frac{1}{2}\left(\left(x+\sqrt{x^{2}-1}\right)^{n}+\left(x-\sqrt{x^{2}-1}\right)^{n}\right), \quad|x| \geq 1,
\end{aligned}
$$

and for the Chebyshev polynomials of the second kind $U_{0}(x)=1, U_{1}(x)=2 x, U_{n+1}(x)=$ $2 x U_{n}(x)-U_{n-1}(x)$ if we put $p(x)=x, q(x)=1, \lambda_{1}(x)=x+\sqrt{x^{2}-1}, \lambda_{2}(x)=x-\sqrt{x^{2}-1}$, $P_{0}(x)=1$ and $P_{1}(x)=2 x$ :

$$
\begin{aligned}
U_{n}(x) & =\frac{1}{\lambda_{1}-\lambda_{2}}\left[\left(\lambda_{1}^{n}-\lambda_{2}^{n}\right) 2 x-\left(\lambda_{1}^{n-1}-\lambda_{2}^{n-1}\right)\right] \\
& =\frac{1}{\lambda_{1}-\lambda_{2}}\left[\lambda_{1}^{n}\left(2 x-\frac{1}{\lambda_{1}}\right)-\lambda_{2}^{n}\left(2 x-\frac{1}{\lambda_{2}}\right)\right] \\
& =\frac{1}{\lambda_{1}-\lambda_{2}}\left(\lambda_{1}^{n+1}-\lambda_{2}^{n+1}\right) \\
& =\frac{1}{2 \sqrt{x^{2}-1}}\left(\left(x+\sqrt{x^{2}-1}\right)^{n+1}-\left(x-\sqrt{x^{2}-1}\right)^{n+1}\right), \quad|x| \geq 1 .
\end{aligned}
$$

Proceeding as above we get the next two formulae for the Chebyshev polynomials of the third and the fourth kind $V_{n}, W_{n}$, respectively:

$$
\begin{aligned}
V_{n}(x) & =\frac{1}{\lambda_{1}-\lambda_{2}}\left[\left(\lambda_{1}^{n}-\lambda_{2}^{n}\right)(2 x-1)-\left(\lambda_{1}^{n-1}-\lambda_{2}^{n-1}\right)\right] \\
& =\frac{1}{\lambda_{1}-\lambda_{2}}\left[\lambda_{1}^{n}\left(2 x-1-\frac{1}{\lambda_{1}}\right)-\lambda_{2}^{n}\left(2 x-1-\frac{1}{\lambda_{2}}\right)\right] \\
& =\frac{1}{\lambda_{1}-\lambda_{2}}\left(\lambda_{1}^{n+1}-\lambda_{2}^{n+1}\right)-\frac{1}{\lambda_{1}-\lambda_{2}}\left(\lambda_{1}^{n}-\lambda_{2}^{n}\right) \\
& =U_{n}(x)-U_{n-1}(x)
\end{aligned}
$$


and

$$
\begin{aligned}
W_{n}(x) & =\frac{1}{\lambda_{1}-\lambda_{2}}\left[\left(\lambda_{1}^{n}-\lambda_{2}^{n}\right)(2 x+1)-\left(\lambda_{1}^{n-1}-\lambda_{2}^{n-1}\right)\right] \\
& =\frac{1}{\lambda_{1}-\lambda_{2}}\left[\lambda_{1}^{n}\left(2 x+1-\frac{1}{\lambda_{1}}\right)-\lambda_{2}^{n}\left(2 x+1-\frac{1}{\lambda_{2}}\right)\right] \\
& =\frac{1}{\lambda_{1}-\lambda_{2}}\left(\lambda_{1}^{n+1}-\lambda_{2}^{n+1}\right)+\frac{1}{\lambda_{1}-\lambda_{2}}\left(\lambda_{1}^{n}-\lambda_{2}^{n}\right) \\
& =U_{n}(x)+U_{n-1}(x)
\end{aligned}
$$

Remark 1.3 If $\operatorname{deg} q=0$, i.e. $q$ is a nonzero constant, one may continue defining polynomials $P_{n}$ for negative integers putting initial polynomials $P_{0}, P_{1}$ arbitrarily and the recurrence formula $P_{n-1}=-\frac{1}{q} P_{n+1}+\frac{2 p}{q} P_{n}$ equivalent to the relation $P_{n+1}=2 p P_{n}(z)-q P_{n-1}$. Same as before we have

$$
\left[\begin{array}{cc}
P_{n} & P_{n-1} \\
P_{n-1} & P_{n-2}
\end{array}\right]=\left[\begin{array}{cc}
2 p & -q \\
1 & 0
\end{array}\right]^{-1}\left[\begin{array}{cc}
P_{n+1} & P_{n} \\
P_{n} & P_{n-1}
\end{array}\right]
$$

as

$$
\left[\begin{array}{cc}
P_{n+1} & P_{n} \\
P_{n} & P_{n-1}
\end{array}\right]=\left[\begin{array}{cc}
2 p & -q \\
1 & 0
\end{array}\right]\left[\begin{array}{cc}
P_{n+1} & P_{n} \\
P_{n} & P_{n-1}
\end{array}\right]
$$

We obtain

$$
\left[\begin{array}{cc}
P_{0} & P_{-1} \\
P_{-1} & P_{-2}
\end{array}\right]=\left[\begin{array}{cc}
2 p & -q \\
1 & 0
\end{array}\right]^{-1}\left[\begin{array}{cc}
P_{1} & P_{0} \\
P_{0} & P_{-1}
\end{array}\right]
$$

and

$$
\begin{aligned}
{\left[\begin{array}{cc}
P_{-n+1} & P_{-n} \\
P_{-n} & P_{-n-1}
\end{array}\right] } & =\left[\begin{array}{cc}
2 p & -q \\
1 & 0
\end{array}\right]^{-n}\left[\begin{array}{cc}
P_{1} & P_{0} \\
P_{0} & P_{-1}
\end{array}\right] \\
& =\frac{1}{\lambda_{1}-\lambda_{2}}\left[\begin{array}{cc}
\lambda_{1} & \lambda_{2} \\
1 & 1
\end{array}\right]\left[\begin{array}{cc}
\lambda_{1}^{-n} & 0 \\
0 & \lambda_{2}^{-n}
\end{array}\right]\left[\begin{array}{cc}
1 & -\lambda_{2} \\
-1 & \lambda_{1}
\end{array}\right]\left[\begin{array}{cc}
P_{1} & P_{0} \\
P_{0} & P_{-1}
\end{array}\right]
\end{aligned}
$$

Multiplying the above matrices we get an analogous formula as (5) in Theorem 1.1:

$$
P_{-n}=\frac{1}{\lambda_{1}-\lambda_{2}}\left[\left(\lambda_{1}^{-n}-\lambda_{2}^{-n}\right) P_{1}-\lambda_{1} \lambda_{2}\left(\lambda_{1}^{-n-1}-\lambda_{2}^{-n-1}\right) P_{0}\right]
$$

Calculating the determinant of the matrix

$$
\left[\begin{array}{cc}
P_{n+1} & P_{n} \\
P_{n} & P_{n-1}
\end{array}\right]=\frac{1}{\lambda_{1}-\lambda_{2}}\left[\begin{array}{cc}
\lambda_{1} & \lambda_{2} \\
1 & 1
\end{array}\right]\left[\begin{array}{cc}
\lambda_{1}^{n-1} & 0 \\
0 & \lambda_{2}^{n-1}
\end{array}\right]\left[\begin{array}{cc}
1 & -\lambda_{2} \\
-1 & \lambda_{1}
\end{array}\right]\left[\begin{array}{cc}
P_{2} & P_{1} \\
P_{1} & P_{0}
\end{array}\right]
$$

we get the Cassini type identity for the polynomials $P_{n}$, corresponding to the Cassini identity for the Fibonacci sequence $F_{n+1} F_{n-1}-F_{n}^{2}=\operatorname{det}\left[\begin{array}{ll}1 & 1 \\ 1 & 0\end{array}\right]^{n}=(-1)^{n}$ :

$$
\begin{aligned}
\left(\lambda_{1}-\lambda_{2}\right)^{2} \operatorname{det}\left[\begin{array}{cc}
P_{n+1} & P_{n} \\
P_{n} & P_{n-1}
\end{array}\right] & =\operatorname{det}\left[\begin{array}{cc}
\lambda_{1} & \lambda_{2} \\
1 & 1
\end{array}\right] \operatorname{det}\left[\begin{array}{cc}
\lambda_{1}^{n-1} & 0 \\
0 & \lambda_{2}^{n-1}
\end{array}\right] \operatorname{det}\left[\begin{array}{cc}
1 & -\lambda_{2} \\
-1 & \lambda_{1}
\end{array}\right] \operatorname{det}\left[\begin{array}{ll}
P_{2} & P_{1} \\
P_{1} & P_{0}
\end{array}\right] \\
\operatorname{det}\left[\begin{array}{cc}
P_{n+1} & P_{n} \\
P_{n} & P_{n-1}
\end{array}\right] & =\operatorname{det}\left[\begin{array}{cc}
\lambda_{1}^{n-1} & 0 \\
0 & \lambda_{2}^{n-1}
\end{array}\right] \operatorname{det}\left[\begin{array}{ll}
P_{2} & P_{1} \\
P_{1} & P_{0}
\end{array}\right]
\end{aligned}
$$


Since $\lambda_{1} \lambda_{2}=q$ we get the following remark.

Remark 1.4 The Cassini type identity for the polynomials $P_{n}$ defined by (1) holds:

$$
P_{n+1} P_{n-1}-P_{n}^{2}=q^{n-1}\left(P_{2} P_{0}-P_{1}^{2}\right)
$$

which implies the four known formulae for the Chebyshev polynomials of the first, second, third and fourth kind, respectively:

$$
\begin{array}{lrr}
T_{n+1}(x) T_{n-1}(x) & -T_{n}^{2}(x)= & x^{2}-1 \\
U_{n+1}(x) U_{n-1}(x) & -U_{n}^{2}(x)= & -1 \\
V_{n+1}(x) V_{n-1}(x) & -V_{n}^{2}(x)= & -2 x-2 \\
W_{n+1}(x) W_{n-1}(x) & -W_{n}^{2}(x)= & 2 x-2 .
\end{array}
$$

Theorem 1.5 Let $P_{n}$ be the sequence of polynomials defined by (1). The quotient $P_{n+1} / P_{n}$ converges uniformly on compact subsets of the set

$$
\left\{z \in \mathbb{C}:\left|\frac{\lambda_{1}(z)}{\lambda_{2}(z)}\right|>1\right\}
$$

to the limit $\lambda_{1}$. The limit does not depend on the initial polynomials $P_{0}$ and $P_{1}$.

Proof. By (5) the quotient of polynomials $P_{n+1}$ and $P_{n}$ equals

$$
\begin{aligned}
\frac{P_{n+1}}{P_{n}} & =\frac{\left(\lambda_{1}^{n+1}-\lambda_{2}^{n+1}\right) P_{1}-\lambda_{1} \lambda_{2}\left(\lambda_{1}^{n}-\lambda_{2}^{n}\right) P_{0}}{\left(\lambda_{1}^{n}-\lambda_{2}^{n}\right) P_{1}-\lambda_{1} \lambda_{2}\left(\lambda_{1}^{n-1}-\lambda_{2}^{n-1}\right) P_{0}} \\
& =\frac{\left(\lambda_{1}-\lambda_{2}\left(\lambda_{2} / \lambda_{1}\right)^{n}\right) P_{1}-\lambda_{1} \lambda_{2}\left(1-\left(\lambda_{2} / \lambda_{1}\right)^{n}\right) P_{0}}{\left(1-\left(\lambda_{2} / \lambda_{1}\right)^{n}\right) P_{1}-\lambda_{2}\left(1-\left(\lambda_{2} / \lambda_{1}\right)^{n-1}\right) P_{0}}
\end{aligned}
$$

It converges on compact subesets the set $\left\{z \in \mathbb{C}:\left|\frac{\lambda_{1}(z)}{\lambda_{2}(z)}\right|>1\right\}$ uniformly to the limit

$$
\frac{\lambda_{1} P_{1}-\lambda_{1} \lambda_{2} P_{0}}{P_{1}-\lambda_{2} P_{0}}=\lambda_{1}
$$

that is independent of $P_{0}$ and $P_{1}$.

\section{Continued fractions related to polynomials $P_{n}$}

Consider the infinite continued fraction

$$
f(z)=2 p(z)-\frac{q(z)}{2 p(z)-\frac{q(z)}{2 p(z)-\ldots}}
$$


and the rational functions $r_{k}$ related to $f$ :

$$
\begin{array}{ccc}
r_{1}=P_{1} / P_{0} & & \\
r_{2}=P_{2} / P_{1} & =\frac{2 p P_{1}-q P_{0}}{P_{1}} & =2 p-\frac{q}{P_{1} / P_{0}} \\
r_{3}=P_{3} / P_{2} & =\frac{2 p P_{2}-q P_{1}}{P_{2}} & =2 p-\frac{q}{P_{2} / P_{1}} \\
\cdots & \cdots \cdots & \cdots \\
r_{n+1}=P_{n+1} / P_{n} & =\frac{2 p P_{n}-q P_{n-1}}{P_{n-1}} & =2 p-\frac{q}{P_{n} / P_{n-1}}
\end{array}
$$

It is easy to see that the function $f$ is the limit of the sequence $r_{k}$.

Theorem 2.1 For arbitrary polynomials $p$ and $q$ such that $\operatorname{deg} p>\operatorname{deg} q>0, q \neq 0$, the continued fraction

$$
f(z)=2 p(z)-\frac{q(z)}{2 p(z)-\frac{q(z)}{2 p(z)-\ldots}}
$$

is a holomorphic function on the set $\left\{z \in \mathbb{C}:\left|\frac{\lambda_{1}(z)}{\lambda_{2}(z)}\right|>1\right\}$ where $\lambda_{1}$ and $\lambda_{2}$ are eigenvalues (4) of the matrix (3).

Proof. The statement follows from Theorem 1.5.

Remark 2.2. In the simple case $p(z)=2 \alpha z$ and $q(z)=\beta^{2}$ the set $\left\{z \in \mathbb{C}:\left|\frac{\lambda_{1}(z)}{\lambda_{2}(z)}\right|>1\right\}$ is the exterior of the interval connecting two points on the complex plane: $\frac{\beta}{\alpha}$ and $-\frac{\beta}{\alpha}$. See Figure 1 for the density plot of the absolute value of the function $r_{60}$ for $p(z)=2 z$ and $q(z)=i=\left(\frac{1+i}{\sqrt{2}}\right)^{2}$ with a visible scar connecting the points $-\frac{1+i}{\sqrt{2}}$ and $\frac{1+i}{\sqrt{2}}$. The following plots exhibit the density plot of $\left|r_{60}\right|$ and more complex scars containing points where the sequence $r_{k}$ is divergent if $p(z)=z^{2}+\frac{i}{10}, q(z)=i$ (Figure 2), $p(z)=i z^{3}+\frac{1}{2}, q(z)=-\frac{1}{3}$ (Figure 3$), p(z)=i z^{2}-\frac{1}{5} z+\frac{1}{10}, q(z)=\left(i-\frac{1}{3}\right) z+\frac{i}{7}+\frac{1}{5}$ (Figure 4$)$.

The plots were created using Mathematica Wolfram Research program.

\section{References}

[1] Donald E. Knuth, The Art of Computer Programming, Addison Wesley, 2nd edition, 1973.

[2] John C. Mason, David Handscomb, Chebyshev polynomials, Chapman \& Hall, 2003. 


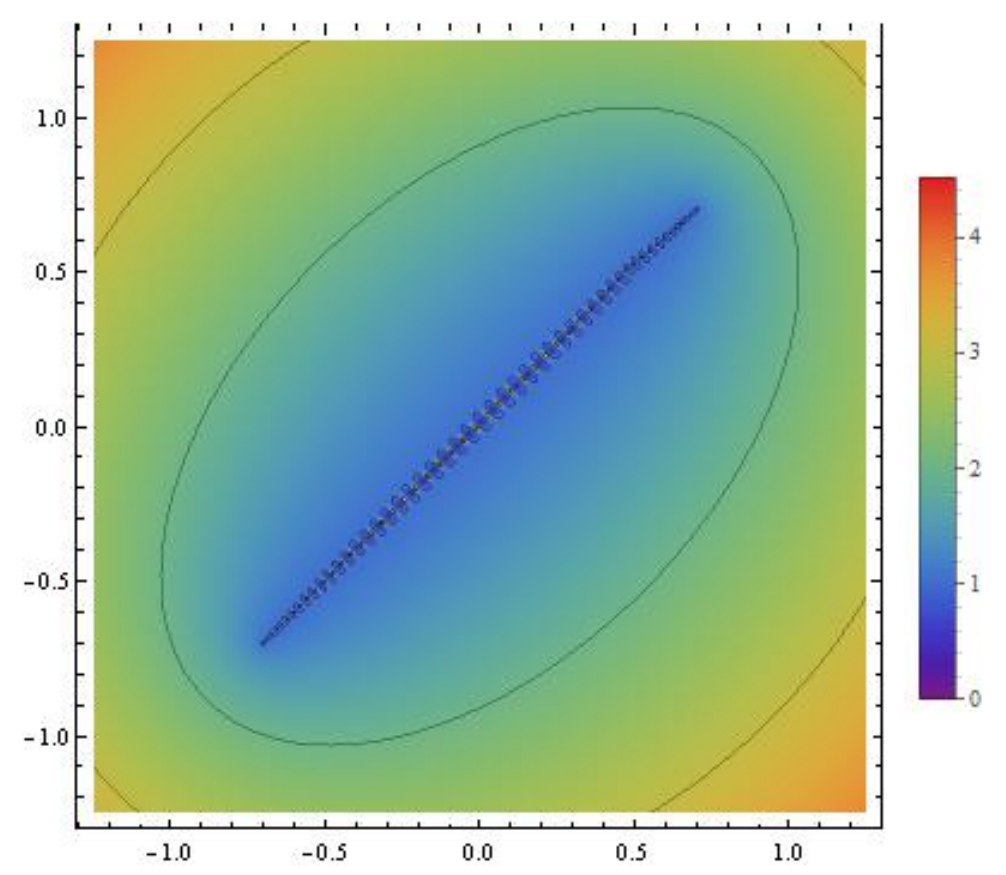

Figure 1: Density plot of $\left|r_{60}\right|$ for $p(z)=2 z$ and $q(z)=i$.

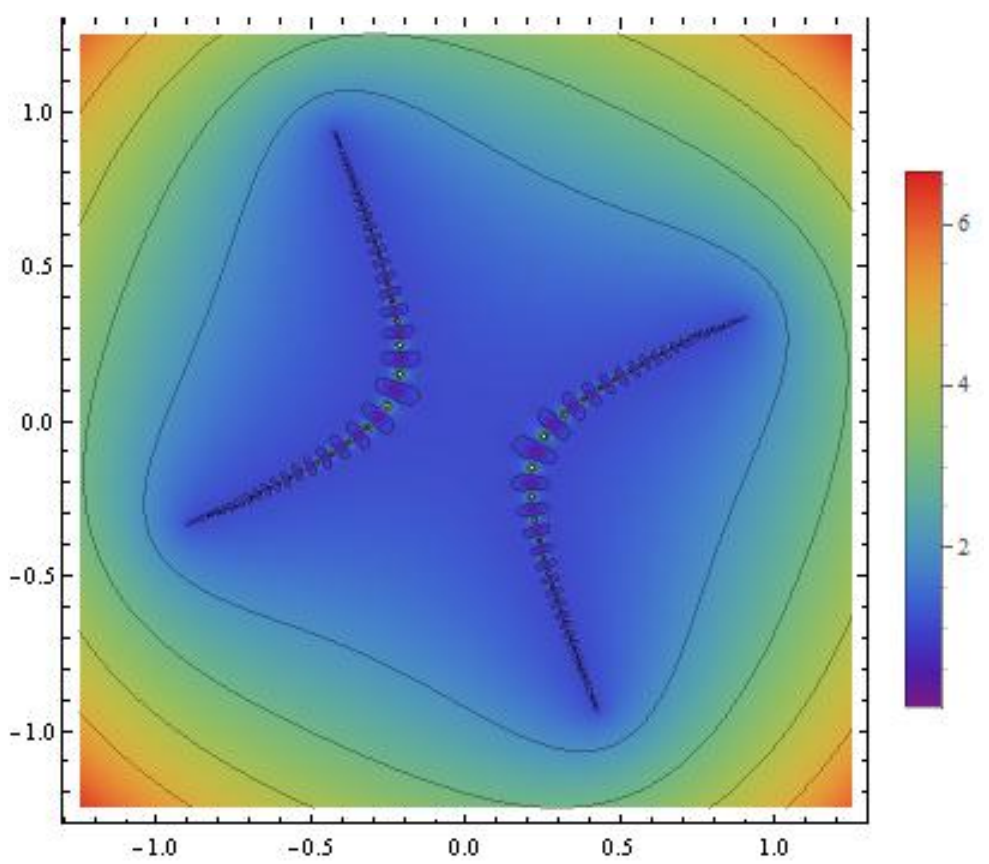

Figure 2: Density plot of $\left|r_{60}\right|$ for $p(z)=z^{2}+\frac{i}{10}, q(z)=i$. 


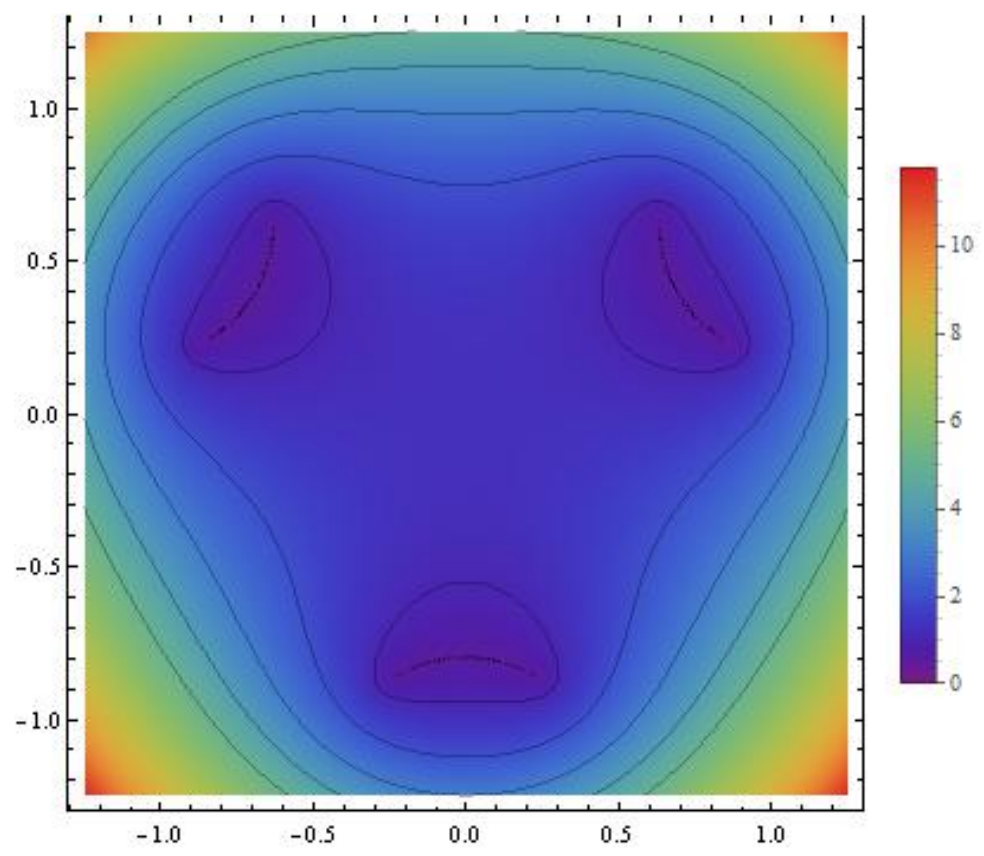

Figure 3: Density plot of $\left|r_{60}\right|$ for $p(z)=i z^{3}+\frac{1}{2}, q(z)=-\frac{1}{3}$.

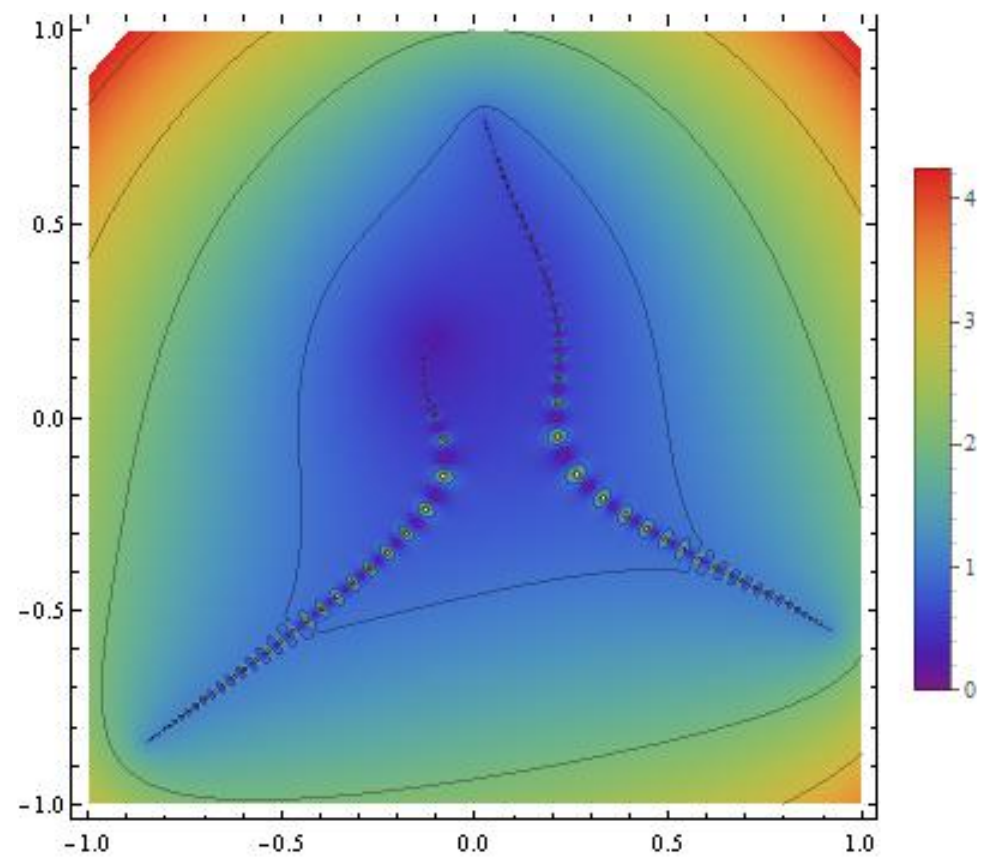

Figure 4: Density plot of $\left|r_{60}\right|$ for $p(z)=i z^{2}-\frac{1}{5} z+\frac{1}{10}, q(z)=\left(i-\frac{1}{3}\right) z+\frac{i}{7}+\frac{1}{5}$. 